\title{
Impact of the NGF Maturation and Degradation Pathway on the Cortical Cholinergic System Phenotype
}

\author{
Simon Allard, ${ }^{1}$ Wanda C. Leon, ${ }^{1}$ Prateep Pakavathkumar, ${ }^{1}$ Martin A. Bruno, ${ }^{4}$ Alfredo Ribeiro-da-Silva, ${ }^{1,2 *}$ \\ and A. Claudio Cuello ${ }^{1,2,3 *}$ \\ ${ }^{1}$ Department of Pharmacology and Therapeutics, McGill University, Montreal, Quebec H3G 1Y6, Canada, ${ }^{2}$ Department of Anatomy and Cell Biology, McGill \\ University, Montreal, Quebec H3A 2B2, Canada, ${ }^{3}$ Department of Neurology and Neurosurgery, McGill University, Montreal, Quebec H3A 2B4, Canada, and \\ ${ }^{4}$ Facultad de Ciencias Médicas, Universidad Católica de Cuyo-CONICET, San Juan, Argentina 5400
}

Cortical cholinergic atrophy plays a significant role in the cognitive loss seen with aging and in Alzheimer's disease (AD), but the mechanisms leading to it remain unresolved. Nerve growth factor (NGF) is the neurotrophin responsible for the phenotypic maintenance of basal forebrain cholinergic neurons in the mature and fully differentiated CNS. In consequence, its implication in cholinergic atrophy has been suspected; however, no mechanistic explanation has been provided. We have previously shown that the precursor of NGF (proNGF) is cleaved extracellularly by plasmin to form mature NGF (mNGF) and that mNGF is degraded by matrix metalloproteinase 9. Using cognitive-behavioral tests, Western blotting, and confocal and electron microscopy, this study demonstrates that a pharmacologically induced chronic failure in extracellular NGF maturation leads to a reduction in mNGF levels, proNGF accumulation, cholinergic degeneration, and cognitive impairment in rats. It also shows that inhibiting NGF degradation increases endogenous levels of the mature neurotrophin and increases the density of cortical cholinergic boutons. Together, the data point to a mechanism explaining cholinergic loss in neurodegenerative conditions such as $\mathrm{AD}$ and provide a potential therapeutic target for the protection or restoration of this $\mathrm{CNS}$ transmitter system in aging and $\mathrm{AD}$.

\section{Introduction}

It is well established that the cortical cholinergic system plays a crucial role in cognitive processing. More precisely, it is involved in attention, learning, and memory formation (Greferath et al., 2000; Veng et al., 2003). Pharmacological evidence suggests that a degeneration of the cholinergic system in normal aged humans and in Alzheimer's patients contributes importantly to cognitive impairment (Perry et al., 1978; Bartus et al., 1982, 1985; Giacobini, 1987; Rogers and Friedhoff, 1996; Gauthier, 1999). Our group has previously demonstrated that the density of cholinergic synapses was significantly decreased in the normal aged rat (Casu et al., 2002) and in animal models of Alzheimer's disease (AD)-like amyloid pathology (Wong et al., 1999; Hu et al., 2003; Bell et al., 2006). As the cortical cholinergic system depends on nerve growth factor (NGF) for its maintenance and the preserva-

\footnotetext{
Received March 3, 2011; revised Dec. 13, 2011; accepted Dec. 16, 2011.

Author contributions: S.A., W.C.L., M.A.B., A.R.-d.S., and A.C.C. designed research; S.A. and P.P. performed research; S.A. and P.P. analyzed data; S.A., A.R.-d.S., and A.C.C. wrote the paper.

This work was supported by Canadian Institutes of Health Research Grants MOP-97776 to A.C.C. and MOP-79411 to A.R.S. A.C.C. is the holder of the McGill University Charles E. Frosst Merck Chair of Pharmacology. A.C.C. is grateful for the unrestricted support from Merck Canada and Dr. Alan Frosst and the Frosst family. We are grateful to Johanne Ouellette for her help with the ultrastructural work; to Manon St-Louis and Adriana Ducatenzeiler for technical assistance; and to Sid Parkinson and Kresimir Krnjevic for editorial assistance.

${ }^{*}$ A.R.-d.S. and A.C.C. contributed equally to this study.

The authors declare no competing financial interests.

Correspondence should be sent to Dr. A. Claudio Cuello, McGill University, Faculty of Medicine, Department of Pharmacology and Therapeutics, 3655 Sir-William 0sler Promenade, \#1210, Montreal, QC H3G 1Y6, Canada. E-mail: claudio.cuello@mcgill.ca.

DOI:10.1523/JNEUROSCI.1144-11.2012

Copyright $\odot 2012$ the authors $\quad 0270-6474 / 12 / 322002-11 \$ 15.00 / 0$
}

tion of cortical cholinergic synapses (Ebendal, 1992; Hefti et al., 1993; Cuello et al., 1994), we questioned whether alterations in the metabolic processing of endogenous levels of cortical NGF would be sufficient to induce a remodeling of cortical cholinergic boutons.

Indeed, recent data from our laboratory demonstrated that, contrary to what has been assumed, NGF is released in an activity-dependent manner in its precursor form, proNGF, in the cortical extracellular space, along with the convertases and proteases necessary to cleave it to its mature form ( $\mathrm{mNGF}$ ) and to degrade the free, unbound mNGF (Bruno and Cuello, 2006). Plasmin was found to be the key serine protease involved in the physiological maturation of proNGF and matrix metalloproteinase 9 (MMP-9), the main protease degrading mNGF (Fig. 1). The marked atrophy of the NGF-dependent forebrain cholinergic system observed in $\mathrm{AD}$ is paradoxically accompanied by normal levels of NGF mRNA (Goedert et al., 1986) and increased levels of proNGF (Fahnestock et al., 2001). Recently, our laboratory has demonstrated that decreased levels of plasmin and increased levels of MMP-9 paralleled the accumulation of proNGF seen in $\mathrm{AD}$ brains. This finding indicates an altered metabolism of NGF in this disease in which the observed elevation of proNGF would be due to a failure of its conversion to mNGF, aggravated by an exacerbated NGF degradation (Bruno et al., 2006, 2009; Cuello and Bruno, 2007). These observations provide a strong link between the status of the NGF metabolic pathway and the well documented cholinergic atrophy (Bowen et al., 1976; Davies and Maloney, 1976). However, no clear demonstration has been made that an alteration in the NGF maturation cascade has a 


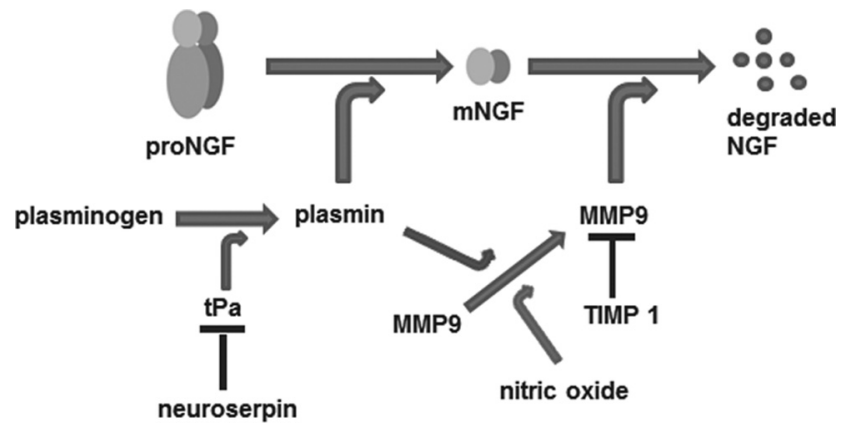

Figure 1. Schematic representation of NGF's extracellular metabolism (modified from Bruno and Cuello, 2006). ProNGF is secreted in the extracellular space along with plasminogen, $\mathrm{tPa}$, and proMMP-9. Plasminogen is cleaved to plasmin by tissue plasminogen activator (tPA), and plasmin cleaves proNGF to $\mathrm{mNGF}$, which can bind to its cognate receptors p75 and TrkA. ProMMP-9 is cleaved by plasmin or nitric oxide to MMP-9, which rapidly degrades the free, unbound $\mathrm{mNGF}$. Neuroserpin is the endogenous inhibitor of tPA and tissue inhibitor of metalloproteinases 1 (TIMP 1) is the endogenous inhibitor of MMP-9.

direct impact on the structure and function of the cortical cholinergic system. In this report, we show that a chronic inhibition of proNGF's extracellular maturation in the medial prefrontal cortex (mPFC) of normal adult rats is sufficient to produce a local atrophy of the cortical cholinergic system paralleled by cognitive impairment. On the other hand, the blockade of the NGF-degrading protease resulted in an increase in cortical cholinergic bouton density.

\section{Materials and Methods}

Fifty adult male Wistar rats weighing $350 \pm 25 \mathrm{~g}$ were studied. All procedures were approved by the McGill University Animal Care Committee and followed the guidelines of the Canadian Council on Animal Care. Three cohorts of animals were used, as follows: cohort 1 (20 rats), to investigate the effects of $\alpha_{2}$-antiplasmin or matrix metalloproteinase 2 and 9 inhibitor (MMP2-9I) treatment on cognitive behavior and cortical synapses; cohort 2 (22 rats), to characterize the effects of each treatment on cortical neurotrophins; and cohort 3 (8 rats), to investigate the effects of $\alpha_{2}$-antiplasmin treatment on the ultrastructural characteristics of cholinergic boutons. The optimal doses and treatment duration were based on results from our previous pilot studies.

\section{Surgery}

Cohort 1. Bilateral cannulae for osmotic pumps (Plastics One), with a lateral cannula spacing of $2 \mathrm{~mm}$ and cut $2.5 \mathrm{~mm}$ below pedestal, were connected with vinyl tubing to osmotic pumps (model 2002, Alzet) that were filled with different solutions under sterile conditions. For the $\alpha_{2}$ antiplasmin experiment, the pumps contained either saline $[0.05 \mathrm{M}$ $\mathrm{NaC}_{2} \mathrm{H}_{3} \mathrm{O}_{2}$ (EMD) and $0.1 \mathrm{M} \mathrm{NaCl}$ (EMD)] or saline and $\alpha_{2}$-antiplasmin (Molecular Innovations), which was dissolved to a concentration of 0.31 $\mu \mathrm{g} / \mu \mathrm{l}$. For the MMP2-9I experiment, the pumps were filled with either vehicle $\left[0.05 \mathrm{M} \mathrm{NaC}_{2} \mathrm{H}_{3} \mathrm{O}_{2}\right.$ and $0.1 \mathrm{M} \mathrm{NaCl}+1 \% \mathrm{DMSO}$ (Sigma)] or vehicle and MMP2-9I type 1 (Calbiochem/EMD), which was dissolved at a concentration of $0.2 \mu \mathrm{g} / \mu \mathrm{l}$. This inhibitor selectively inhibits matrix metalloproteinases 2 and 9 over other matrix metalloproteinases. To properly dissolve the inhibitor, the solution was sonicated three times for $5 \mathrm{~s}$ and cooled between intervals by putting it on ice for $30 \mathrm{~s}$. All solutions were sterilized using a $0.2 \mu \mathrm{m}$ syringe filter (Nalgene), and the pumps were filled under sterile conditions in a bio-safety cabinet. Before surgery, the animals were anesthetized with isoflurane, put in a stereotaxic apparatus (Stoelting), and kept deeply anesthetized using a nose adaptor for isoflurane anesthesia (Harvard Apparatus). Details about the surgical procedure have been provided previously (Hu et al., 1997). The bilateral cannulae (Plastics One) were implanted at the following coordinates: anteroposterior $+2.2 \mathrm{~mm}$, lateral \pm 1 relative to bregma, reaching a depth of $2.5 \mathrm{~mm}$ below the skull to target the mPFC (Fig. 2 B). All coordinates were derived from the Paxinos and Watson rat brain atlas (Paxinos and Watson, 2005).
Cohorts 2 and 3 . The surgery was done in the same way, except that a 28 gauge unilateral cannula replaced the bilateral cannula. The cannulae were also cut $2.5 \mathrm{~mm}$ below the pedestal and implanted at the following coordinates: anteroposterior $+2.2 \mathrm{~mm}$, lateral +1 .

\section{Behavioral testing (cohort 1)}

To measure the effects of the treatment on learning, memory consolidation, and memory retrieval, two different water maze protocols were used. Before treatment, the animals were put in the pool three times a day for 6 consecutive days. The animals were given $120 \mathrm{~s}$ to find the hidden platform (position 1), on which they remained for $10 \mathrm{~s}$ before they were taken out of the pool, dried, and put back in their cages. A video camera tracking system was used to record the swimming path (HVS Image). The time required for each rat to reach the platform, defined as latency, and the distance swum during each trial were measured. Following treatment and a $2 \mathrm{~d}$ washout period, the animals were put in the same pool in the absence of the platform (probe test) for $120 \mathrm{~s}$. The video tracking system recorded the number of times the animals crossed the area where the platform had been ( platform passes), and that number was used as an index of spatial memory retrieval. The day following the probe test, the platform was reinserted in the pool at a different location (position 2) and the animals were given six trials of $120 \mathrm{~s}$ to find the platform in the new position in a $1 \mathrm{~d}$ water maze protocol (Leon et al., 2010). The time required to find the platform (latency) was recorded throughout trials as an index of spatial learning. On the following day, the platform was removed and a second probe test was performed. The platform passes at the new location were recorded as an index of memory formation.

\section{Preparation of the brain tissue}

Following treatment, the animals were deeply anesthetized with Equithesin $(6.5 \mathrm{mg}$ of chloral hydrate and $3 \mathrm{mg}$ of sodium pentobarbital in a volume of $0.3 \mathrm{ml}$, i.p., per $100 \mathrm{~g}$ of body weight) and perfused through the left ventricle with cold saline. Each cohort was then processed as follows.

Cohort 1. Brains were removed, and the injection site in one hemisphere was dissected, put in cold RIPA buffer, and homogenized for neurochemical analysis. The region dissected corresponded to a block with a $4 \mathrm{~mm}$ side centered on the cannula trace. The other hemisphere was put in a cold fixative made of $0.1 \mathrm{~m}$ phosphate buffer $(\mathrm{PB})$ containing $4 \%$ formaldehyde obtained from paraformaldehyde (EMD), pH 7.4, and left in the fixative at $4^{\circ} \mathrm{C}$ for $24 \mathrm{~h}$. The solution was then changed to a cryoprotective $30 \%$ sucrose solution made in $0.1 \mathrm{M} \mathrm{PB}$ for another $24 \mathrm{~h}$.

Cohort 2. Brains were removed, and the injection site (a $4 \mathrm{~mm}$ block around the cannula trace) was dissected out and homogenized in cold RIPA buffer. A same-sized block on the contralateral cortex at the same anteroposterior position was also dissected out and processed in the same manner.

Cohort 3. Following perfusion with cold saline for $1 \mathrm{~min}$ to clear blood vessels, the saline was replaced by a fixative solution containing $4 \%$ formaldehyde, $0.5 \%$ glutaraldehyde, and $15 \%$ picric acid in $0.1 \mathrm{M} \mathrm{PB}, \mathrm{pH} 7.4$. The flow of the fixative was kept high for $1 \mathrm{~min}$ and was reduced dropwise for $30 \mathrm{~min}$. After this first fixation, the fixative solution was replaced by a second fixative solution containing $4 \%$ formaldehyde and $15 \%$ picric acid in $0.1 \mathrm{M} \mathrm{PB}$.

\section{Neurochemical analysis of neurotrophins}

NGF and BDNF levels were measured using SDS/PAGE and Western blotting of the cortical homogenates. The amount of protein was measured using a conventional protein assay protocol (Bio-Rad Laboratories) using BSA (Fisher Scientific Canada) as a standard. Following protein quantification, proteins were loaded and resolved on a $12 \%$ acrylamide gel. Fifty micrograms of total protein was loaded for the detection of proNGF and mNGF as $15 \mu \mathrm{g}$ was loaded for the detection of proBDNF and mature BDNF (mBDNF). The proteins were transferred to a PVDF membrane with a wet transfer system (Bio-Rad Laboratories). The membranes were washed with Tris-Buffered Saline + Tween 20 (Invitrogen Canada) (TBS+T), blocked for $1 \mathrm{~h}$ in $5 \%$ nonfat milk in TBS $+\mathrm{T}$. The membranes were then incubated with the primary antibody (rabbit anti-NGF or rabbit anti-BDNF diluted 1:1000; Santa Cruz Biotechnology) in $5 \%$ milk in TBS $+\mathrm{T}$ overnight at $4^{\circ} \mathrm{C}$. The following day, the membranes were washed in $\mathrm{TBS}+\mathrm{T}$ and incubated with the second- 
ary antibody (horseradish peroxidase conjugated goat anti-rabbit IgG diluted 1:5000; Jackson ImmunoResearch) in $5 \%$ milk in TBS $+\mathrm{T}$ for $1 \mathrm{~h}$. Before detection, the membranes blotted for proNGF and mNGF were cut horizontally at the height of the $25 \mathrm{kDa}$ marker, and the top half containing proNGF was detected differently than the bottom half containing mNGF. The membranes were washed with TBS $+\mathrm{T}$, and the immunoreactive bands corresponding to proBDNF, mBDNF, and proNGF were visualized with ECL (GE Healthcare Life Sciences) using the Kodak Biomax XAR imaging film kit with exposure times from 1 to $2 \mathrm{~min}$. The membranes with mNGF were visualized with the ECL plus reagent (GE Healthcare Life Sciences) and exposed for $10 \mathrm{~min}$. The membranes were washed in TBS $+\mathrm{T}$ and incubated with another primary antibody (mouse anti $\beta$-tubulin, diluted 1:50 000; Sigma) with 5\% milk in TBS $+\mathrm{T}$ for $1 \mathrm{~h}$. The membranes were washed with $\mathrm{TBS}+\mathrm{T}$ and then incubated with the secondary antibody (horseradish peroxidase-conjugated goat anti-mouse antibody diluted 1:5000; Jackson ImmunoResearch) with $5 \%$ milk in TBS+T. Following wash in TBS $+\mathrm{T}$, the signal was detected as described above. The films were digitized using a light box and video camera attached to an MCID M4 image analysis system (Imaging Research Inc.), and the optical density of individual bands was measured with the MCID software. The optical density of NGFor BDNF-related bands was always divided by the intensity of the tubulin band from the same sample to obtain the relative optical density. All Western blots were performed in duplicate. Differences between averages from treated and vehicle-treated groups were analyzed with a Student's $t$ test.

Tissue preparation for confocal microscopy

Details concerning the antibodies used for each type of immunolabeling are found in Table 1. Following cryoprotection in 30\% sucrose in $0.1 \mathrm{M}$ phosphate buffer, the brains were embedded in an OCT compound (Somagen), frozen, and cut in the coronal plane at a thickness of $35 \mu \mathrm{m}$ with a cryostat. The sections containing $\mathrm{mPFC}$ were recovered in PBS, treated in $50 \%$ ethanol for $20 \mathrm{~min}$, and washed $3 \times 10 \mathrm{~min}$ in PBS. The sections were blocked in a $10 \%$ normal serum solution of the animal species of the secondary antibody in PBS $+\mathrm{T}$ for $1 \mathrm{~h}$ and then incubated in the primary antibody diluted in a 5\% normal serum in PBS $+\mathrm{T}$ overnight at $4^{\circ} \mathrm{C}$. Five different immunolabelings were performed on alternate sections to characterize the effects of the treatments on many neurotransmitter systems. Four different single labelings were performed with antibodies directed against the vesicular acetylcholine transporter (VAChT), the vesicular glutamate transporter 1 (VGluT1), glutamic acid decarboxylase 65 (GAD 65), and neurotrophic tyrosine kinase receptor type 1 (TrkA) to label cholinergic, glutamatergic, and GABAergic boutons and the high-affinity NGF receptor TrkA, respectively. An additional double labeling was performed with antibodies directed against tyrosine hydroxylase (TH) and dopamine $\beta$-hydroxylase (DBH) to detect and differentiate dopaminergic and noradrenergic boutons. All labelings were balanced so that there was no experimental bias where one neurotransmitter system was analyzed with sections closer to the infusion point. The following day, the sections were washed $3 \times 10 \mathrm{~min}$ in PBS, and then were incubated in a solution containing the appropriate secondary antibody and 5\% normal serum in PBS. The sections were degradation.

\section{B}

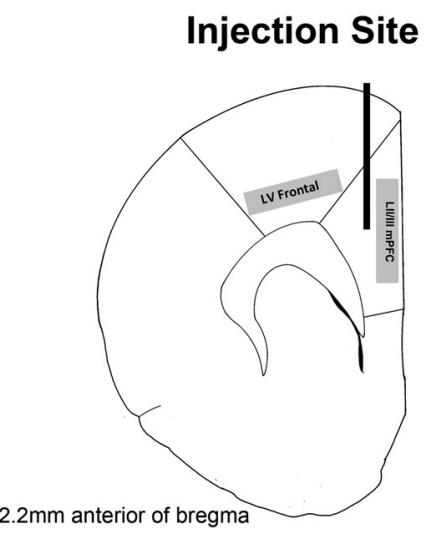

D

MMP2-9 inhibitor infusions

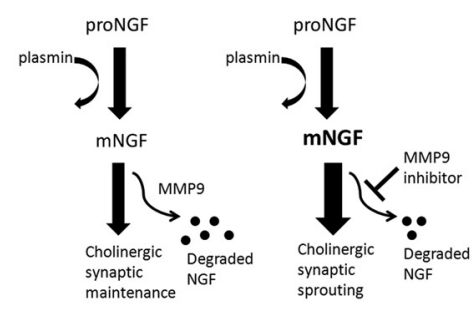

Figure 2. Schematic representation of the treatment strategy. $\boldsymbol{A}$, Drug treatment duration (broken lines in $\boldsymbol{a}$ and $\boldsymbol{b}$ ) to assess its effects on neurotrophin levels $(\boldsymbol{a})$ and to assess the resulting behavioral outcomes and synaptic remodeling after the washout period $(\boldsymbol{b})$. $\boldsymbol{B}$, Cortical cannulae were implanted in the $\mathrm{mPFC}$, and the synaptic densities were measured in the treatment area and in the adjacent frontal cortex to establish anatomical specificity of the observed synaptic alterations (gray boxes). $\boldsymbol{C}, \alpha_{2}-$ antiplasmin was used to inhibit the conversion of proNGF to mNGF. D, A synthetic MMP2-9 inhibitor was used to inhibit mNGF

washed $3 \times 10 \mathrm{~min}$ in PBS, mounted on gelatin subbed slides, dried overnight at $4^{\circ} \mathrm{C}$, and coverslipped with Aqua Polymount (Polysciences).

\section{Confocal microscopy and quantification}

Images were taken on a Zeiss LSM 510 confocal microscope equipped with Ar and He-Ne lasers (Carl Zeiss Canada), using a $63 \times$ PlanApochromatic oil-immersion objective and the Zeiss LSM software. For each rat, four sections per immunolabel were sampled with five images per section acquired. All images, which represented confocal optical sections $\sim 0.5 \mu \mathrm{m}$ in thickness, were taken in laminae II/III of the mPFC. They were always taken at a distance between 0.2 and $1.5 \mathrm{~mm}$ from the cannula path. To avoid experimental bias, the observer was blinded to the identity of the experimental groups. Each confocal image corresponded to a field of $146.3 \times 146.3 \mu \mathrm{m}$ for the VAChT and TH+DBH labeling and $73.2 \mu \mathrm{m} \times 72.2 \mu \mathrm{m}$ for the GAD 65 and VGluT1 labelings. The images were exported as TIFF files to be analyzed with an image analysis system (MCID Elite version 7, Imaging Research Inc). In short, the immunoreactive elements were isolated from the background with a brightness threshold, and size and shape criteria were used to count varicosities. As opposed to other labelings that were enriched in varicosities, TrkA labeling decorated axons and varicosities. For overall brightness measurements, the brightness of all immunoreactive material above the minimum brightness threshold was measured. For the count of TrkAimmunoreactive (IR) varicosities, size and shape criteria were applied to separate varicosities from axons. Confocal settings for acquisition and quantification were as previously described (Majdi et al., 2007; Allard et al., 2011). Differences between groups (vehicle vs treated) were analyzed using Student's $t$ tests. 
Table 1. Antibodies used for the labeling of cortical synaptic boutons

\begin{tabular}{|c|c|c|c|}
\hline Anatomical marker & Primary antibody & $\begin{array}{l}\text { Serum used for blocking step } \\
\text { and antibody dilutions }\end{array}$ & Secondary antibody \\
\hline VAChT (expressed in cholinergic varicosities) & $\begin{array}{l}\text { Goat anti-VAChT; 1:10,000 (Millipore), } \\
\text { polyclonal }\end{array}$ & $\begin{array}{l}\text { Normal rabbit serum (Jackson } \\
\text { ImmunoResearch) }\end{array}$ & Biotinylated rabbit anti-goat; 1:200 (Vector Labs) \\
\hline $\begin{array}{l}\text { TH (expressed in both dopaminergic and } \\
\text { noradrenergic varicosities) }\end{array}$ & Rabbit anti-TH; 1:3000 (Millipore), polyclonal & $\begin{array}{l}\text { Normal goat serum (Jackson } \\
\text { ImmunoResearch) }\end{array}$ & $\begin{array}{l}\text { Alexa Fluor 488-conjugated goat anti-rabbit; 1:800 } \\
\text { (Invitrogen) }\end{array}$ \\
\hline DBH (expressed in noradrenergic varicosities) & Mouse anti-DBH; 1:25 (MediMabs), monoclonal & $\begin{array}{l}\text { Normal donkey serum (Jackson } \\
\text { ImmunoResearch) }\end{array}$ & $\begin{array}{l}\text { Rhodamine Red X-conjugated donkey anti-mouse; 1:200 } \\
\text { (Jackson ImmunoResearch) }\end{array}$ \\
\hline VGluT1 (expressed in glutamatergic varicosities) & $\begin{array}{l}\text { Mouse anti-VGluT1; 1:100 (characterized in Bell } \\
\text { et al. (2006); MediMabs), monoclonal }\end{array}$ & $\begin{array}{l}\text { Normal donkey serum (Jackson } \\
\text { ImmunoResearch) }\end{array}$ & $\begin{array}{l}\text { Alexa Fluro 488-conjugated donkey anti-mouse; 1:800 } \\
\text { (Invitrogen) }\end{array}$ \\
\hline GAD 65 (expressed in GABAergic varicosities) & $\begin{array}{l}\text { Mouse anti-GAD-65; 1:1000 (Millipore), } \\
\text { monoclonal }\end{array}$ & $\begin{array}{l}\text { Normal donkey serum (Jackson } \\
\text { ImmunoResearch) }\end{array}$ & $\begin{array}{l}\text { Alexa Fluor 488-conjugated donkey anti-mouse; 1:800 } \\
\text { (Invitrogen) }\end{array}$ \\
\hline $\begin{array}{l}\text { Neurotrophic TrkA high-affinity mNGF receptor } \\
\text { (expressed on cholinergic axon) }\end{array}$ & Polyclonal goat anti-TrkA; 1:100 (R\&D Systems) & $\begin{array}{l}\text { Normal donkey serum (Jackson } \\
\text { ImmunoResearch) }\end{array}$ & $\begin{array}{l}\text { Rhodamine Red X-conjugated donkey anti-goat; 1:200 } \\
\text { (Jackson ImmunoResearch) }\end{array}$ \\
\hline
\end{tabular}

\section{Tissue preparation for electron microscopy}

Fixed brains were cut in 50- $\mu$ m-thick coronal sections with a vibrating microtome (Vibratome 1000 plus). Sections containing the mPFC were collected in PBS. The sections adjacent to the cannula tract were incubated for $1 \mathrm{~h}$ in $30 \%$ sucrose and $10 \%$ glycerol followed by $1 \mathrm{~h}$ in $30 \%$ sucrose and 20\% glycerol for cryoprotection. The individual sections were snap frozen and thawed three times in liquid nitrogen-cooled isopentane to permeabilize the tissue. The sections were then recovered in PBS, washed in PBS for $3 \times 10 \mathrm{~min}$, and then incubated in $1 \%$ sodium borohydride in PBS for 30 min followed by extensive washings with PBS until all the bubbles disappeared. Subsequently, the sections were placed in a solution of $10 \%$ normal rabbit serum in PBS for $1 \mathrm{~h}$ and incubated overnight at $4^{\circ} \mathrm{C}$ with a goat anti-VAChT antibody (1:10,000; Millipore) in 5\% normal rabbit serum in PBS. The following day, the sections were washed $3 \times 10 \mathrm{~min}$ in PBS then incubated in a biotinylated rabbit antigoat secondary antibody (1:200; Vector Labs) in 5\% normal rabbit serum in PBS. See Table 1 for details of the antibodies used. The sections were washed $3 \times 10$ min in PBS and then incubated in the A and B solution (VECTASTAIN Elite ABC kit, Vector Labs) for $1 \mathrm{~h}$. The sections were further washed $3 \times 10 \mathrm{~min}$ in PBS, and incubated in DAB (Sigma) at a concentration of $0.5 \mathrm{mg} / \mathrm{ml}$ in PBS containing $1 \%$ cobalt chloride and 1\% nickel ammonium sulfate for 15 min (Ribeiro-da-Silva et al., 1993). Hydrogen peroxide was then added to the wells to make a final concentration of $0.01 \%$ to initiate the enzymatic reaction. The reaction was terminated after $10 \mathrm{~min}$ by the addition of an excess of PBS. The sections were washed $3 \times 10 \mathrm{~min}$ in PBS and transferred to glass vials containing $0.1 \mathrm{M}$ PB. The sections were postfixed in $1 \%$ osmium tetroxide in $0.1 \mathrm{M} \mathrm{PB}$ for $1 \mathrm{~h}$ at $4^{\circ} \mathrm{C}$. After washing, the sections were dehydrated in ascending ethanol solutions followed by propylene oxide, flat embedded in Epon between a plastic coverslip and acetate foil, and cured in the oven for $24 \mathrm{~h}$ at $60^{\circ} \mathrm{C}$, as described in detail previously (Ribeiro-da-Silva et al., 1993). After re-embedding, the Epon blocks were trimmed so that the sections to be cut were $\sim 1 \mathrm{~mm}^{2}$ in area, covering mostly laminae II and III of the $\mathrm{mPFC}$ at a distance between 0.5 and $1.5 \mathrm{~mm}$ from the cannula site. Semithin and ultrathin sections were cut using an ultramicrotome (Ultracut III, Reichert-Jung). The ultrathin sections were mounted on single-slot copper grids coated with Formvar, contrast stained with uranyl acetate and lead citrate, and observed with a Philips 410 LS electron microscope. Digital images were obtained with a Megaview II digital camera (Olympus Soft Imaging Solutions), and saved in the TIFF format.

\section{Analysis of electron micrographs}

In EM micrographs, the cross-sectional area of VAChT-IR elements was measured with the help of the MCID Elite image analysis software. Parameters were established to enable the software to autonomously detect VAChT-positive immunoreactivity. The total area covered by the immunolabeling in each micrograph was measured in square micrometers. The proportion of immunoreactive elements (axonal boutons) forming classical synapses in the plane of the section was manually counted from the photographs. The immunoreactive boutons were considered as synaptic when they had an electron-dense presynaptic density and they were ap- posed to a dendrite; a synaptic cleft and a thin postsynaptic density had to be visible as well, as illustrated in Figure 6A, $B$. Differences between average cross-sectional areas were analyzed by means of Student's $t$ tests. To verify whether the changes in the percentage of boutons that made classical synapses were significant, the individual percentages were compared after arcsine conversion using a $t$ test. The observer was blinded to the experimental groups.

\section{Results}

\section{proNGF and BDNF levels during and after $\alpha_{2}$-antiplasmin or} MMP2-9I treatment

Following Western blotting, the pro and mature forms of NGF and BDNF were identified based on their molecular weights, where proNGF migrated close to $40 \mathrm{kDa}$, proBDNF migrated at $\sim 35 \mathrm{kDa}$, and both mature neurotrophins migrated at $\sim 14 \mathrm{kDa}$. The described bands are consistent with other publications using the same antibodies on rat tissue (Bruno and Cuello, 2006; Ullal et al., 2007) and aligned with bands from positive controls (mouse submandibular gland extracts for NGF and transfected SYHY cells for BDNF) (data not shown). Consistent with our previous findings (Bruno and Cuello, 2006), we demonstrated that infusions of $\alpha_{2}$-antiplasmin led to the significant accumulation of proNGF $(p=0.025)$ and a significant reduction of $\mathrm{mNGF}$ $(p=0.016)$ in the mPFC at 2 weeks (Fig. 3A). Conversely, the infusion of an MMP2-9I led to the accumulation of mNGF ( $p=$ 0.018 ) and no significant change in proNGF levels (Fig. $3 B$ ). The levels of BDNF (proBDNF and mBDNF), with this dose and regime, were not affected significantly by either treatment (Fig. $3 A, B)$. Importantly, following treatment, washout and behavioral analysis (cohort1), the levels of all neurotrophins were comparable between experimental groups (see Figs. 5, 7). For the $\alpha_{2}$-antiplasmin experiment, the relative optical density of treated animals compared with saline was of 0.51 versus 0.56 for proNGF $(p=0.77), 0.032$ versus 0.028 for $\operatorname{mNGF}(p=0.86), 0.62$ versus 0.66 for proBDNF $(p=0.71)$, and 0.068 versus 0.059 for mBDNF $(p=0.76)$. For the MMP2-9I experiment, the relative optical density of treated animals compared with saline was of 0.73 versus 0.68 for proNGF ( $p=0.74), 0.054$ versus 0.050 for $\mathrm{mNGF}$ $(p=0.87), 0.64$ versus 0.61 for proBDNF $(p=0.85)$, and 0.056 versus 0.053 for $\operatorname{mBDNF}(p=0.78)$.

\section{Learning and memory parameters after preventing NGF} conversion with infusions of $\alpha_{2}$-antiplasmin into the mPFC To establish whether a prolonged inhibition of NGF maturation resulted in a measurable cognitive impairment, we tested $\alpha_{2}$ antiplasmin- and saline-injected animals using a modified ver- 
sion of the Morris water maze. The neural networks recruited when the animals are performing in the water maze are complex, and the mPFC is not involved in all cognitive aspects of the task. For this particular reason, we elaborated a variant of the classical Morris water maze test (Morris et al., 1982) that allows us to separate the effects of the treatment on spatial learning, memory retrieval, and memory consolidation (Leon et al., 2010). To elucidate the role of the $\mathrm{mPFC}$ cholinergic system in the different phases of the water maze paradigm, the animals were trained and tested for memory retrieval at strategic times before and after the treatment (Fig. $4 A$, diagram). As expected, all animals showed a typical and comparable learning curve before the treatment (Fig. 4B). Groups to receive either $\alpha_{2}$-antiplasmin or saline were randomly formed after this initial testing, and the average escape latency on the 2 last days for the groups was compared (data not shown). We saw no difference in the baseline performance of the two randomly formed groups, emphasizing that group differences following treatment should reflect a direct consequence of the pharmacological manipulation. Following drug infusions restricted to the mPFC, both groups performed similarly on the probe test to measure the retention of the spatial memory of the platform in position 1 (Fig. 4C). These data suggest that the treatment did not impair long-term memory retrieval. The treatment did not affect subsequent learning either, as both groups displayed similar post-treatment learning curves for the platform in position 2 (Fig. 4D). On the other hand, when the two groups were tested on the following day for retention of the new platform position, $\alpha_{2}$-antiplasmin-treated animals had significantly fewer platform passes than vehicletreated animals $(p=0.02)$ (Fig. $4 E)$. These results indicate that the animals treated with $\alpha_{2}$-antiplasmin were impaired in consolidating the new memory of the platform in position 2, which is consistent with the role of the $\mathrm{mPFC}$ in the consolidation of recent memories (Leon et al., 2010).

\section{Neurotransmitter-specific presynaptic densities following pharmacological interference with the conversion of proNGF to $\mathrm{mNGF}$ in the $\mathrm{mPFC}$}

After treatment ( $\alpha_{2}$-antiplasmin or saline) for 2 weeks, we investigated the relative density of transmitter-specific presynaptic boutons using antibodies directed against VAChT, TH, DBH, VGluT1, and GAD65 to label cholinergic, dopaminergic, noradrenergic, glutamatergic, and GABAergic boutons, respectively. At the 2 week time point, we also observed a significant reduction in the density of cholinergic boutons (average of 2425 boutons per field in saline-treated animals and 997 in $\alpha_{2}$-antiplasmin-treated animals; $p=0.0008$ ), whereas the other bouton populations were unaffected (Fig. $5 A$ ). As TrkA NGF receptors are expressed on cortical cholinergic terminals (Sobreviela et al., 1994), we labeled and quantified TrkA receptors in the mPFC. These observations revealed a significant decrease in the numbers of TrkA-IR structures in $\alpha_{2}$-antiplasmin-treated animals when compared with saline-treated ones (average of 784 boutons per field in saline-treated animals and 585 in $\alpha_{2}$-antiplasmin-treated animals; $p=0.03$ ). To estimate the average receptor level per TrkA-IR structure, we kept confocal settings (lasers and digital gain) constant for all images. We used an MCID image analysis system to measure the average brightness of the immunoreactive elements. A trend toward a decrease in the average brightness of TrkA-IR boutons from $\alpha_{2}$-antiplasmin-treated rats was observed (average of 0.7 relative brightness in saline-treated animals and 0.4 in $\alpha_{2}$ antiplasmin-treated animals; $p=0.06$ ), suggesting that the remaining cholinergic presynaptic boutons expressed a reduced number of TrkA receptors (Fig. 5B). To establish whether the effect of the treatment was limited to the area of the mPFC close to the site of injection and to better interpret the behavioral changes induced by the pharmacological treatment, we quantified the density of VAChT-IR boutons in lamina $\mathrm{V}$ of the adjacent frontal cortex (Fig. $2 B$ ) and found no significant change in bouton density (average of 1538 boutons per field in saline-treated animals and 1409 in $\alpha_{2}$-antiplasmintreated animals; $p=0.7)$. We treated another cohort of animals with the same pharmacological protocol and processed the MPFC tissue for electron microscopy. The ultrastructural data revealed that VAChT-IR boutons from $\alpha_{2}$-antiplasmin-treated animals became atrophic and lost synaptic contacts. Using three-dimensional reconstructions, our laboratory has previously demonstrated that most of the cholinergic boutons in lamina $\mathrm{V}$ of the rat parietal cortex formed classical synapses on pyramidal neurons (Turrini et al., 2001). Using electron micrographs taken from a single ultrathin section, we found that $55 \%$ of the cholinergic boutons in lamina III of the 


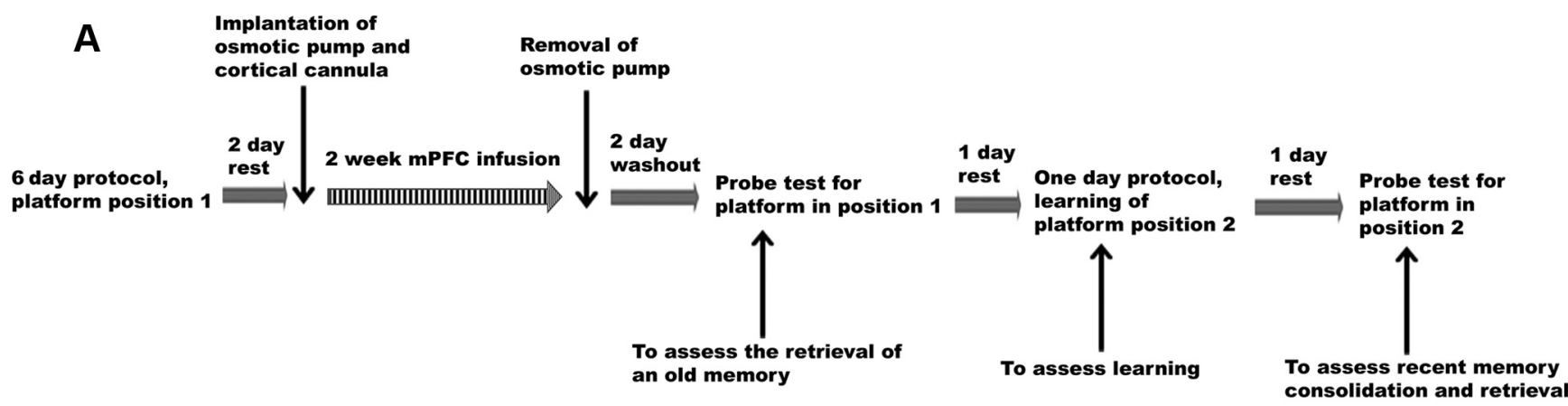

B 6 day learning, platform in position 1

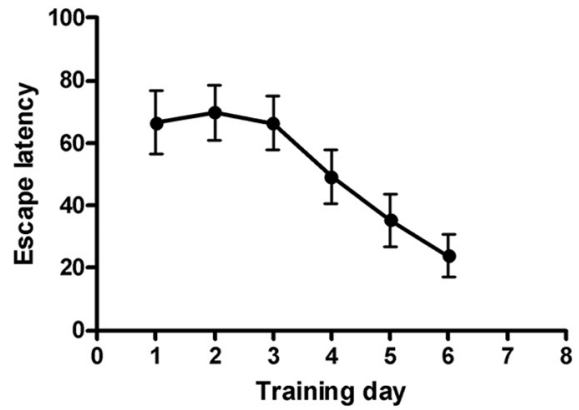

D

One day learning, platform in position 2

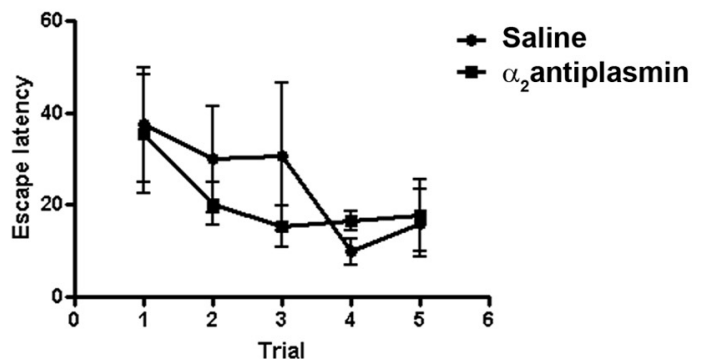

C

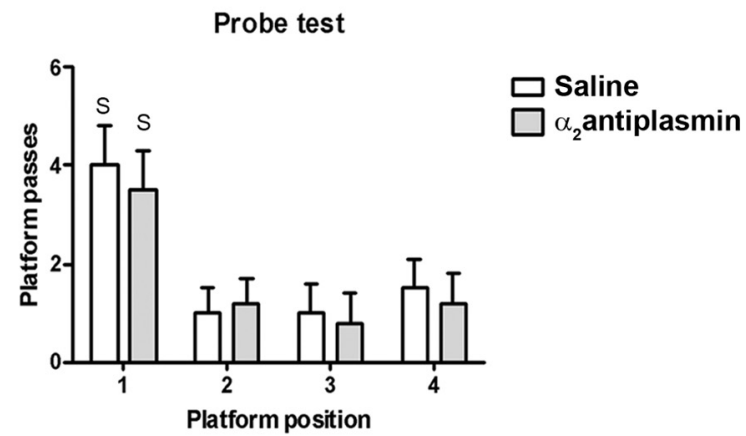

$\mathbf{E}$

Probe test

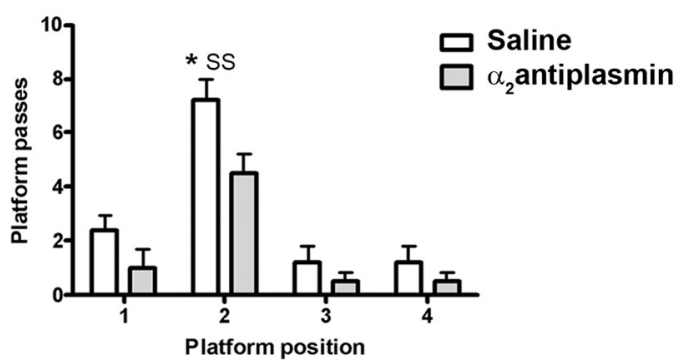

Figure 4. Behavioral consequences of $\alpha_{2}$-antiplasmin-induced cholinergic atrophy. $A$, Before treatment, the rats received $6 \mathrm{~d}$ of water maze training with the platform in position 1 . The 2 week treatment was initiated following a $2 \mathrm{~d}$ rest and consolidation period. Following treatment and a washout period of $2 \mathrm{~d}$, a probe test was performed to characterize the effects of the synaptic remodeling on old memory retrieval. Following the probe test, the animals were trained again with the platform in position 2 . On the following day, a second probe test was performed to characterize the effects of the induced synaptic remodeling on recent memory consolidation and retrieval. $\boldsymbol{B}$, Learning curve of all the animals before the implantation of osmotic pumps. ( $n=10)$. $\boldsymbol{C}$, Probe test $1: 2 \mathrm{~d}$ after the removal of the osmotic pumps, the animals were put back in the pool in the absence of the platform. The number of passes where the platform used to be was recorded as an index of spatial memory. The passes at other putative platform locations were also recorded as an index of locomotor activity ( $n=5$ per group) ( $S p<0.05$ when compared with other platform passes from the same group, Tukey's test). D, Learning curve of the animals trained with a $1 \mathrm{~d}$ learning protocol (Leon et al., 2010) where the platform was placed in position 2 . Note that the $x$-axis indicates individual trials, as opposed to averages per day. E, Probe test 2 was performed on the day following the $1 \mathrm{~d}$ learning protocol ( ${ }^{*} p<0.05$, when saline and $\alpha_{2}$-antiplasmin are compared for platform $2 ; S S p<0.01$, when compared with other platform passes from the same group by Tukey's test).

medial prefrontal cortex formed synaptic appositions (Fig. 6). Following $\alpha_{2}$-antiplasmin treatment, only $34 \%$ of the remaining cholinergic boutons were forming classical synapses $(p=0.004)$. The remaining cholinergic varicosities also displayed a significantly smaller cross-sectional area (average of $0.22 \mu \mathrm{m}^{2}$ in salinetreated animals and $0.15 \mu \mathrm{m}^{2}$ in $\alpha_{2}$-antiplasmin-treated animals; $p=0.02$ ) (Fig. 6).

Learning and memory after inhibiting mNGF degradation To test whether a 2 week inhibition of MMP-2 and MMP-9 in a restricted area of the cerebral cortex had behavioral consequences, we used the same experimental plan as the one described for the $\alpha_{2}$-antiplasmin experiments. In contrast to the $\alpha_{2}$-antiplasmin treatment, inhibiting MMP2-9 did not reveal a significant effect on learning, memory formation or retrieval (data not shown).
Neurotransmitter-specific synaptic densities following pharmacological interference with the degradation of $\mathrm{mNGF}$ in the mPFC

Following behavioral testing, we processed cortical samples for immunocytochemistry using the same antibodies as for the $\alpha_{2}$ antiplasmin experiment. Treated animals had a significantly higher density of cholinergic boutons than vehicle-treated animals (average of 2250 boutons per field in vehicle-treated animals and 2950 in MMP2-9I-treated animals; $p=0.02$ ) (Fig. 7A). The treatment appeared to be specific for the cholinergic system as no change in the density of dopaminergic, noradrenergic, glutamatergic, or GABAergic boutons was observed. We also detected a significant increase in the number of TrkA-IR boutons in MMP2-9I-treated rats (average of 760 boutons per field in vehicle-treated animals and 999 in MMP2-9I-treated animals; $p=0.02$ ). On the other hand, the aver- 

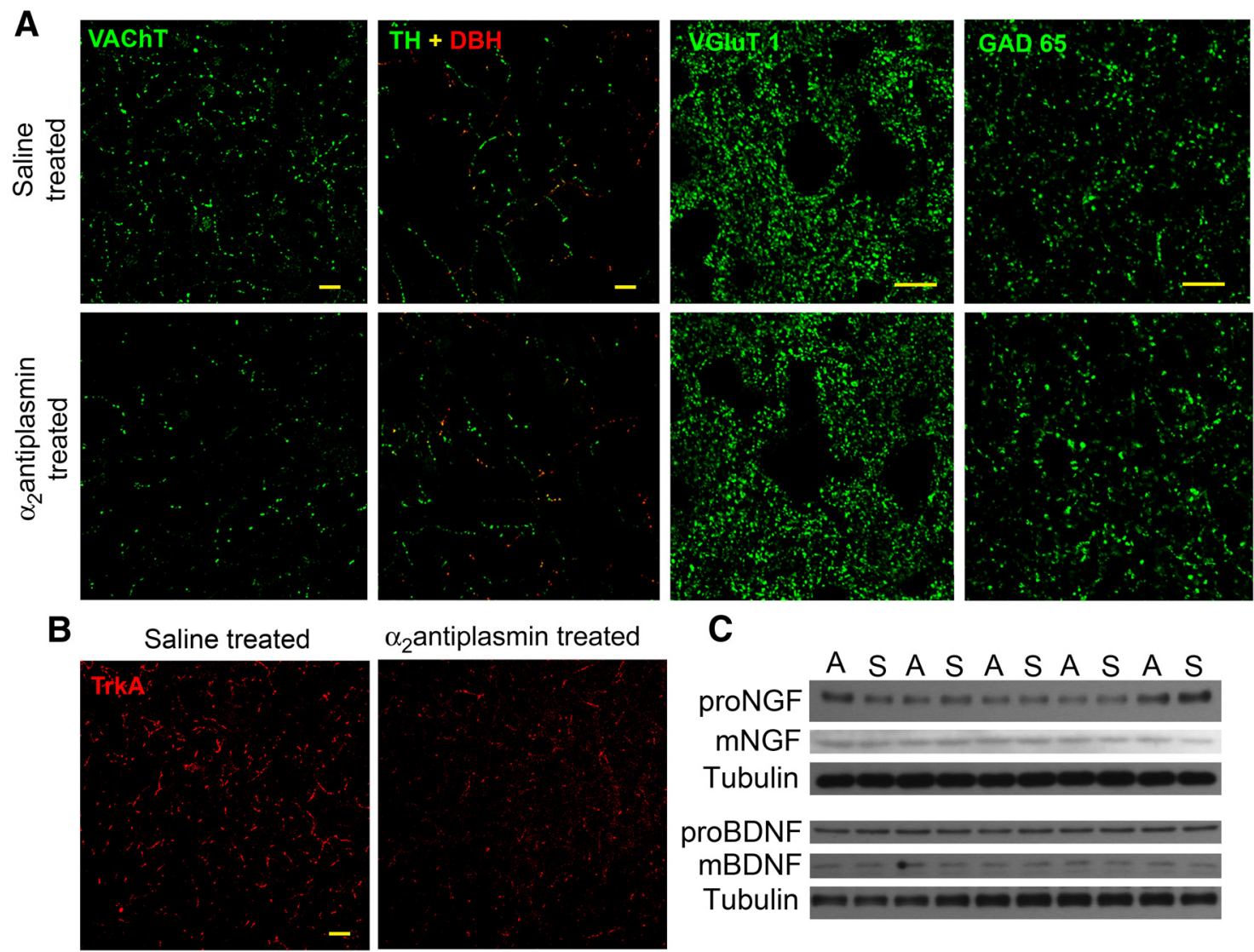

C
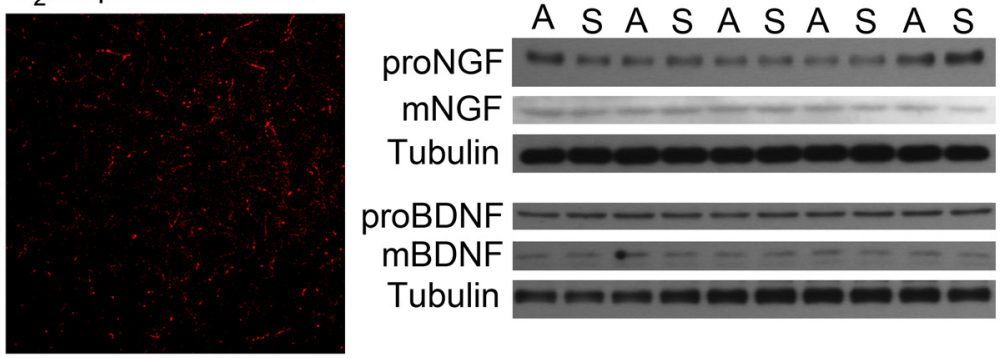

Figure 5. Synaptic remodeling following a 2 week $\alpha_{2}$-antiplasmin infusion. $\boldsymbol{A}$, Representative confocal images of immunostainings performed to characterize quantitatively the treatment-induced neurotransmitter-specific synaptic remodeling. These images represent VAChT-labeled cholinergic boutons, solely TH-labeled dopaminergic boutons, TH- and DBH-labeled noradrenergic boutons, VGluT1labeled glutamatergic boutons, and GAD 65-labeled GABAergic boutons. Note the apparent decrease of VAChT-IR elements in $\alpha_{2}$-antiplasmin-treated animals. Note the specific decrease in VAChT-IR boutons (quantitative data in text). $\boldsymbol{B}$, Representative confocal images and quantification of TrkA-IR elements in saline and $\alpha_{2}$-antiplasmin-treated animals. The $\alpha_{2}$-antiplasmin treatment led to a loss of TrkA-IR elements (quantitative data in text). C, Western blotting indicates unaltered NGF and BDNF levels following washout period and behavioral testing. A, Animals that were formerly treated with $\alpha_{2}$-antiplasmin; $\mathrm{S}$, animals that were formerly treated with saline (quantitative data in text).

age brightness in both treatment groups was indistinguishable, suggesting that the TrkA expression levels per cholinergic axon were conserved (average of 0.65 relative brightness in vehicle-treated animals and 0.55 in MMP2-9I-treated animals; $p=0.7$ ).

\section{Discussion}

This study provides evidence that it is possible to modulate the cortical cholinergic phenotype by interfering with the newly described extracellular metabolism of NGF (Bruno and Cuello, 2006). Thus, inhibition of proNGF conversion led to cholinergic atrophy, which was accompanied by a mild cognitive deficit. In contrast, inhibition of mNGF degradation resulted in the formation of new cholinergic boutons, but no behavior alterations.

\section{Validation of the pharmacological manipulation}

To downregulate or upregulate the endogenous levels of cortical mNGF, we used a constant intracortical infusion of $\alpha_{2}$ antiplasmin or of a synthetic MMP2-9 inhibitor, respectively. Since plasmin can cleave proBDNF released from cultured neurons (Lee et al., 2001) and MMP-9 was also found to cleave it in the kindled hippocampus (Mizoguchi et al., 2011), we quantified the effects of both treatments on BDNF maturation to assess specificity. Our results demonstrated no significant effect of either treatment on BDNF maturation at the dose used. The fact that NGF and BDNF have different kinetics in vivo could explain such differential effects. As both the proBDNF and mBDNF can be released by neurons (Yang et al., 2009), evidence shows that proNGF but not mNGF can be released in the extracellular space (Bruno and Cuello, 2006). Once bound to its activated receptor, internalized, and retrogradely transported (Grimes et al., 1996), NGF is exclusively targeted to the lysosome where it is degraded (Claude et al., 1982; Bernd and Greene, 1983; Butowt and von Bartheld, 2001). BDNF, on the other hand, can be redirected to the Golgi network to be recycled (Butowt and von Bartheld, 2001). Furthermore, BDNF but not NGF, was found to travel anterogradely (Altar et al., 1997; Smith et al., 1997) and undergo transcytosis (von Bartheld et al., 1996), leading to the notion that the mature form of BDNF is used as a "neuronal currency" that can be exchanged between neurons involved in a given circuit (von Bartheld et al., 2001). The more stable nature of BDNF over NGF could explain why, at the dose used, there was a preferential effect over the NGF system. Such a view is also consistent with the fact that both proBDNF and mBDNF can be measured in comparable amounts when cortical tissue is processed for Western blotting. On the other hand, mNGF can only be detected with a very strong protocol, at conditions that lead to a very strong saturation of the proNGF signal. Interestingly, in our hands, the acute injection of much higher doses was capable of reducing cortical mBDNF levels, supporting the dose relationship on the effects on respective neurotrophic systems (data not shown). This would be in line with 

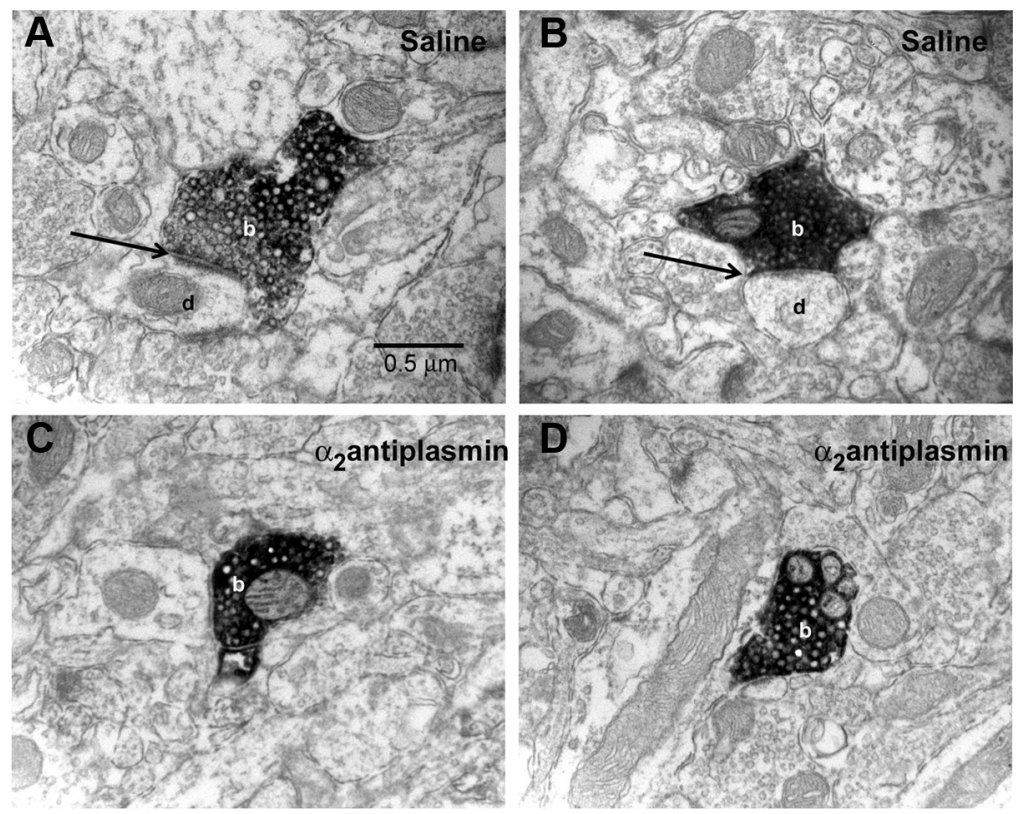

Figure 6. Ultrastructural analysis of cholinergic synapses following $\alpha_{2}$-antiplasmin treatment. Electron micrographs represent typical cholinergic boutons (b) from saline- $(\boldsymbol{A}, \boldsymbol{B})$ and $\alpha_{2}$-antiplasmin-treated $(\boldsymbol{C}, \boldsymbol{D})$ animals. The dark electron dense precipitate indicates VAChT immunoreactivity. The arrows in $\boldsymbol{A}$ and $\boldsymbol{B}$ point at the synaptic cleft of a classical cholinergic synapse. $b, A$ presynaptic cholinergic bouton immunoreactive for VAChT, filled with synaptic vesicles, apposed to a dendritic shaft, and displaying an electron dense presynaptic density; $d$, a dendritic shaft with neurotubules and a thin postsynaptic density. Following quantification (quantitative data in text), it was found that a smaller percentage of immunoreactive boutons were making classical synapses following $\alpha_{2}$-antiplasmin treatment. As well, the average cross sectional area of immunoreactive elements was reduced following $\alpha_{2}$-antiplasmin treatment.

the observation that the total demise of plasmin, as produced by tissue plasminogen activator (tPA) knockout, can interfere with proBDNF cleavage (Pang et al., 2004).

\section{Effects of $\alpha_{2}$-antiplasmin treatment on the steady-state number of cortical synapses}

Morphologically, we observed a decrease in the density of cholinergic boutons, while the dopaminergic, noradrenergic, glutamatergic, and GABAergic bouton counts were unaltered (Fig. 5A). This observation is consistent with the fact that, in the present experimental paradigm, we measured a significant treatment effect only on proNGF and mNGF levels (Fig. $3 A$ ) and that the noncholinergic cortical synaptic populations are not known to depend on NGF signaling. Electron microscopy revealed that $\alpha_{2}-$ antiplasmin treatment led to atrophy and a synaptic disconnection of the remaining cholinergic boutons (Fig. 6). An earlier study from our laboratory showed that a decreased volume of cholinergic boutons accompanied the age-associated cholinergic bouton loss (Turrini et al., 2001). As anatomical and functional evidence suggest that acetylcholine acts both by classical (synaptic) and volume transmission (Turrini et al., 2001; Sarter et al., 2009), we demonstrate that a reduced supply of endogenous mNGF decreases the percentage of cholinergic varicosities forming synapses, likely resulting in impaired cholinergic transmission.

\section{Possible mechanisms behind cholinergic atrophy}

As discussed above, the constant infusion of $\alpha_{2}$-antiplasmin reduced the availability of mNGF and increased the levels of proNGF (Fig. 3A). Since proNGF has been reported to have the potential to induce apoptosis in vitro (Lee et al., 2001; Nykjaer et al., 2004; Volosin et al., 2006), it is possible that the increased amounts of proNGF in the extracellular space could contribute to this atrophy. On the other hand, other groups have reported proNGF to be rather mildly neurotrophic (Fahnestock et al., 2004). We assume that what contributes the most to the observed atrophy is the lack of mNGF signaling, since endogenous mNGF regulates the steady-state number of cholinergic synapses in the cerebral cortex (Debeir et al., 1999). These downstream effects of a lack of mNGF could explain why the remaining cholinergic fibers expressed lower amounts of the NGF receptor following $\alpha_{2}$-antiplasmin treatment (Fig. $5 D, E$ ). More precisely, it has been shown that TrkA expression on CNS cholinergic neurons is dependent on NGF trophic support (Venero et al., 1994; Figueiredo et al., 1995; Williams et al., 2005). Given that proNGF binds preferably to $\mathrm{p} 75$, such a shift in receptor balance could mirror an increased proNGF/mNGF ratio in the target cortical tissue resulting from changes in extracellular metabolism (Bruno and Cuello, 2006). Importantly, both the increase in proNGF and the decrease in TrkA cortical levels are early features of the AD pathology (Counts et al., 2004; Peng et al., 2004). Single-cell RT-PCR on human nucleus basalis neurons revealed decreased TrkA but not p75 mRNA levels in early AD (Mufson et al., 2007), where TrkA receptor levels correlated with premortem cognitive scores.

\section{Effects of cholinergic atrophy on cognitive performance}

Pharmacological evidence suggests that defects in cholinergic transmission contribute to the cognitive deficits observed in normal aging and in $\mathrm{AD}$ (Bartus et al., 1982; Burns et al., 1999). To test whether cholinergic atrophy in the mPFC would have an impact on cognitive function, we applied $\alpha_{2}$-antiplasmin to inhibit the conversion of proNGF to mNGF. The application of $\alpha_{2}$-antiplasmin was restricted to the mPFC and produced a local downregulation of cholinergic boutons, which resulted in impairments in the consolidation of a recent spatial memory. Given that after a washout period, at the time of behavioral testing, the neurotrophic levels returned to normal (Figs. 5, 7), it can confidently be assumed that the behavioral changes reflect the transmitter-specific remodeling rather than effects of altered neurotrophin levels. The absence of cholinergic loss in the adjacent frontal cortex demonstrates that the treatment was anatomically restricted to the mPFC (see Results for quantitative data). The fact that $\alpha_{2}$-antiplasmin-treated rats displayed impairment in the second probe test indicates a failure in the consolidation and/or retrieval of the recently acquired spatial information. Our data are consistent with the effects of scopolamine, which impairs the consolidation and retrieval of recent spatial memories (Huang et al., 2011). Previous investigations have demonstrated that the mPFC's involvement in spatial memory formation was more significant in the consolidation phase than in the acquisition phase (Kraemer et al., 1996; Leon et al., 2010). These observations are consistent with the present finding that induction of 

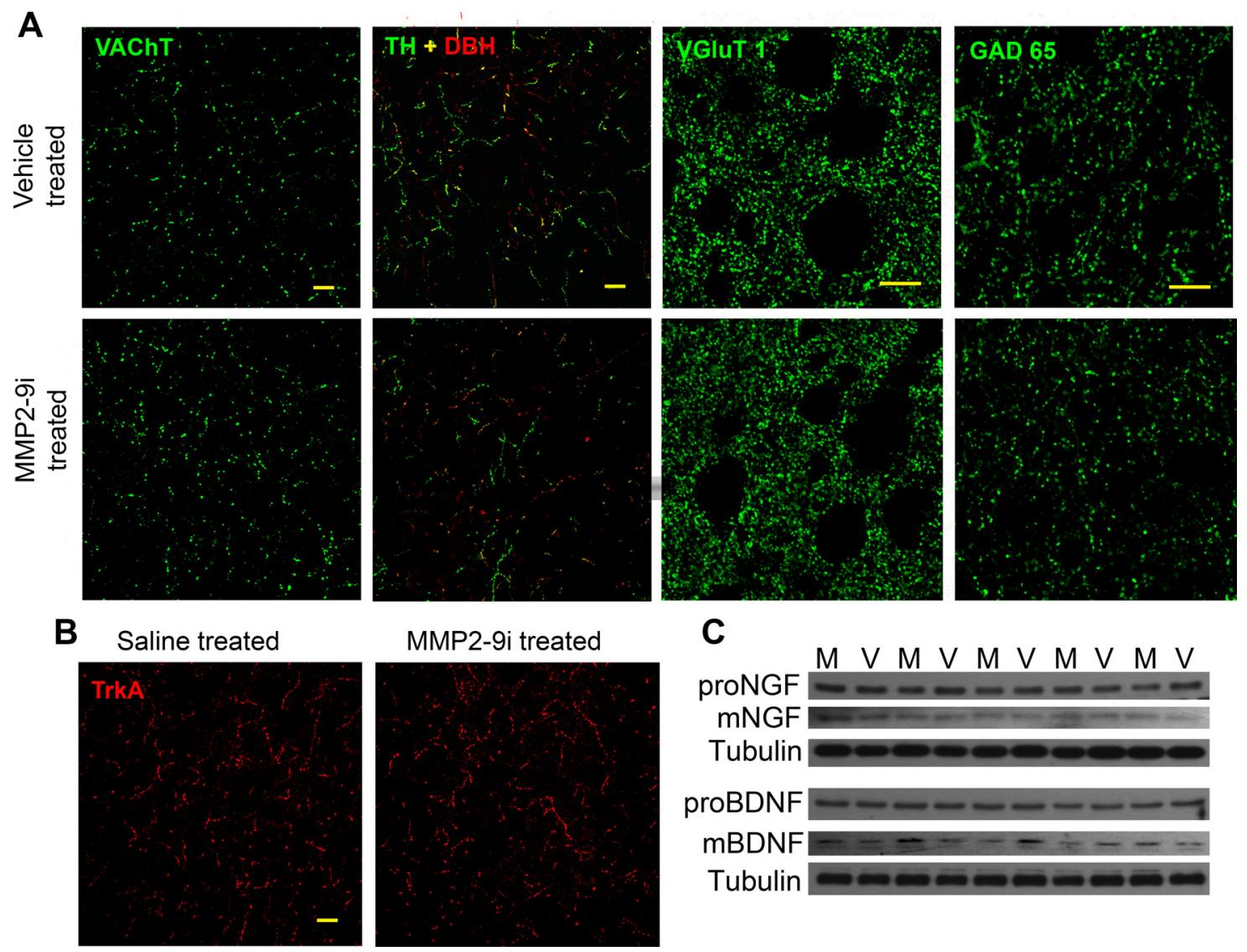

Figure 7. Synaptic remodeling following a 2 week MMP2-9l infusion. A, Representative confocal images of immunostainings performed to characterize quantitatively the treatment-induced neurotransmitter-specific synaptic remodeling. These images represent VAChT-labeled cholinergic boutons, solely TH-labeled dopaminergic boutons, TH- and DBH-labeled noradrenergic boutons, VGluT1labeled glutamatergic boutons, and GAD 65-labeled GABAergic boutons. Note the apparent increase of VAChT-IR elements in MMP2-91-treated animals (quantitative data in text). $\boldsymbol{B}$, Representative confocal images of TrkA-IR elements in vehicle- and MMP2-91-treated animals. The MMP2-91 treatment led to an increase of TrkA-IR elements (quantitative data in text). No change was observed in the average brightness of immunoreactive elements. C, Western blotting indicates unaltered NGF and BDNF levels following the washout period and behavioral testing. M, Animals that were formerly treated with the MMP2-9 inhibitor; $V$, animals that were formerly treated with the vehicle (quantitative data in text).

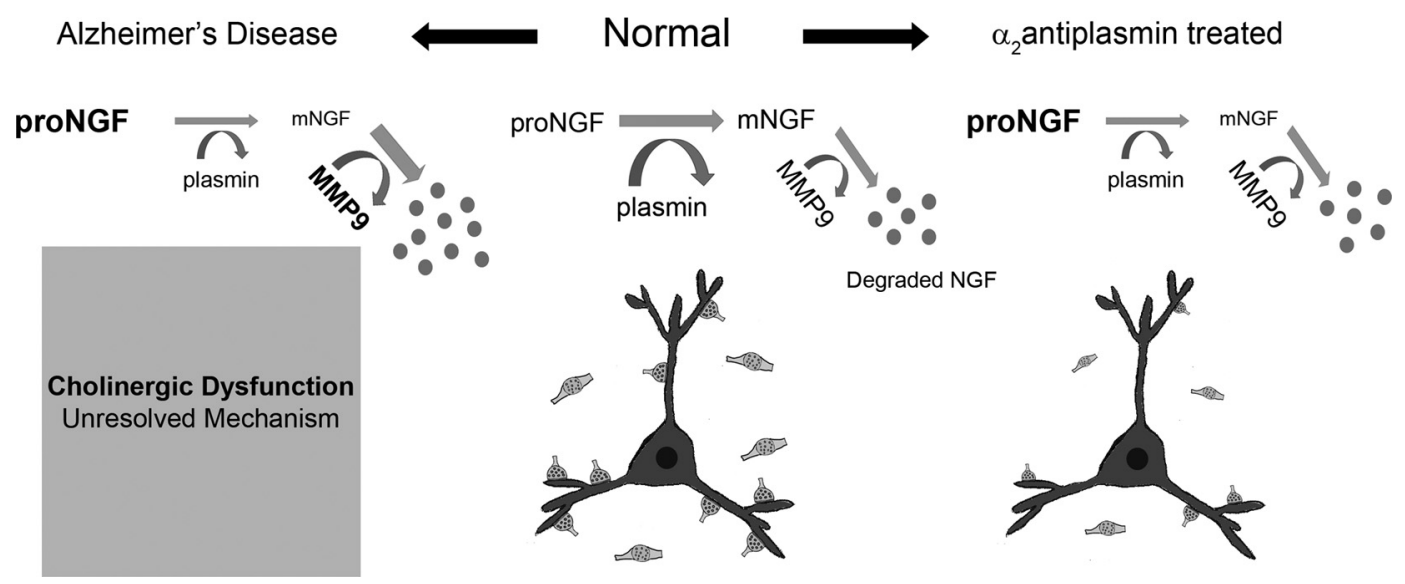

Figure 8. Schematic representation of NGF's extracellular metabolism as a mechanism to maintain the cholinergic phenotype of basal forebrain neurons and explaining cholinergic atrophy in Alzheimer's disease. Neurochemical analysis of human postmortem brains revealed alterations in NGF's extracellular metabolic proteins in AD when compared with age-matched controls (Bruno et al., 2009). These alterations indicated an imbalance leading to decreased available mNGF. To validate whether the proposed NGF metabolic pathway is responsible for the cholinergic phenotype, we pharmacologically inhibited extracellular proNGF maturation in the cortex of normal rats. This inhibition was sufficient to produce a local cholinergic atrophy as measured by a loss of cholinergic boutons, an atrophy of the terminals, and a disruption of their normal synaptic pattern.

cholinergic atrophy in the mPFC does not impair new learning but rather the consolidation of new memories (Fig. 4). The fact that our pharmacologically induced cholinergic atrophy prevented the consolidation and retrieval of a new memory but spared the retrieval of an older memory (consolidated before the treatment) suggests that spatial memories lose their dependence on the mPFC cholinergic system over time. Such a view is also consistent with the effect in humans of scopolamine, which im- 
pairs the encoding of new memories but spares older memories (Atri et al., 2004).

\section{Increasing local availability of $\mathrm{mNGF}$ is capable of upregulating cholinergic synapses}

The fact that the basal level of local cortical cholinergic boutons was increased in normal animals following constant MMP2-9 inhibition is a proof of the principle that cortical cholinergic boutons can be upregulated by increasing the available targetderived neurotrophic support, even in the adult CNS (Fig. 7). In that regard, further experiments will be needed to determine whether such a strategy has a therapeutic value in conditions where the cholinergic system is compromised, such as in normal aging and $\mathrm{AD}$.

\section{Conclusions}

Using a constant delivery of $\alpha_{2}$-antiplasmin in the mPFC of normal rats, we demonstrated that extracellular cleavage of proNGF in the target tissue is necessary for the maintenance of the normal density of cholinergic projections from the basal forebrain. The inhibition of proNGF maturation led to an accumulation of proNGF, a reduction in cortical cholinergic bouton numbers, an atrophy of the cholinergic terminals, and a disruption of the normal synaptic patterns of cholinergic boutons (Fig. 8). The ensuing NGF-trophic disconnect resulted in a synaptic cholinergic-specific lesion accompanied with behavioral impairments, demonstrating that cholinergic transmission in the MPFC is necessary for spatial memory consolidation but not for acquisition or retrieval of old memories. Using a synthetic inhibitor of MMP2-9, we demonstrated that augmenting the available mNGF was sufficient to increase the steady-state number of cortical cholinergic synapses. Such a manipulation might be of therapeutic interest in conditions where a deficit of cholinergic basal forebrain synapses is known to occur, such as in aging and $\mathrm{AD}$.

\section{References}

Allard S, Gosein V, Cuello AC, Ribeiro-da-Silva A (2011) Changes with aging in the dopaminergic and noradrenergic innervation of rat neocortex. Neurobiol Aging 32:2244-2253.

Altar CA, Cai N, Bliven T, Juhasz M, Conner JM, Acheson AL, Lindsay RM, Wiegand SJ (1997) Anterograde transport of brain-derived neurotrophic factor and its role in the brain. Nature 389:856-860.

Atri A, Sherman S, Norman KA, Kirchhoff BA, Nicolas MM, Greicius MD, Cramer SC, Breiter HC, Hasselmo ME, Stern CE (2004) Blockade of central cholinergic receptors impairs new learning and increases proactive interference in a word paired-associate memory task. Behav Neurosci 118:223-236.

Bartus RT, Dean RL 3rd, Beer B, Lippa AS (1982) The cholinergic hypothesis of geriatric memory dysfunction. Science 217:408-414.

Bartus RT, Dean RL, Pontecorvo MJ, Flicker C (1985) The cholinergic hypothesis: a historical overview, current perspective, and future directions. Ann N Y Acad Sci 444:332-358.

Bell KF, Ducatenzeiler A, Ribeiro-da-Silva A, Duff K, Bennett DA, Cuello AC (2006) The amyloid pathology progresses in a neurotransmitter-specific manner. Neurobiol Aging 27:1644-1657.

Bernd P, Greene LA (1983) Electron microscopic radioautographic localization of iodinated nerve growth factor bound to and internalized by PC12 cells. J Neurosci 3:631-643.

Bowen DM, Smith CB, White P, Davison AN (1976) Neurotransmitter related enzymes and indices of hypoxia in senile dementia and other abiotrophies. Brain 99:459-496.

Bruno MA, Cuello AC (2006) Activity-dependent release of precursor nerve growth factor, conversion to mature nerve growth factor, and its degradation by a protease cascade. Proc Natl Acad Sci U S A 103:6735-6740.

Bruno MA, Ravid R, Cuello AC (2006) Altered proNGF maturation and NGF degradation and the vulnerability of forebrain cholinergic neurons in Alzheimer's disease. Alzheimers Dement 2 [Suppl]:S476.

Bruno MA, Leon WC, Fragoso G, Mushynski WE, Almazan G, Cuello AC
(2009) Amyloid beta-induced nerve growth factor dysmetabolism in Alzheimer disease. J Neuropathol Exp Neurol 68:857-869.

Burns A, Rossor M, Hecker J, Gauthier S, Petit H, Möller HJ, Rogers SL, Friedhoff LT (1999) The effects of donepezil in Alzheimer's disease- results from a multinational trial. Dement Geriatr Cogn Disord 10:237-244.

Butowt R, von Bartheld CS (2001) Sorting of internalized neurotrophins into an endocytic transcytosis pathway via the Golgi system: ultrastructural analysis in retinal ganglion cells. J Neurosci 21:8915-8930.

Casu MA, Wong TP, De Koninck Y., Ribeiro-da-Silva A, Cuello AC (2002) Aging causes a preferential loss of cholinergic innervation of characterized neocortical pyramidal neurons. Cereb Cortex 12:329-337.

Claude P, Hawrot E, Parada I (1982) Ultrastructural studies on the intracellular fate of 125I-nerve growth factor in cultured rat sympathetic neurons. J Cell Biochem 20:1-13.

Counts SE, Nadeem M, Wuu J, Ginsberg SD, Saragovi HU, Mufson EJ (2004) Reduction of cortical TrkA but not p75(NTR) protein in early-stage Alzheimer's disease. Ann Neurol 56:520-531.

Cuello AC, Bruno MA (2007) The failure in NGF maturation and its increased degradation as the probable cause for the vulnerability of cholinergic neurons in Alzheimer's disease. Neurochem Res 32:1041-1045.

Cuello AC, Garofalo L, Liberini P, Maysinger D (1994) Cooperative effects of gangliosides on trophic factor-induced neuronal cell recovery and synaptogenesis: studies in rodents and subhuman primates. Prog Brain Res 101:337-355.

Davies P, Maloney AJF (1976) Selective loss of central cholinergic neurons in Alzheimer's disease. Lancet 2:1403.

Debeir T, Saragovi HU, Cuello AC (1999) A nerve growth factor mimetic TrkA antagonist causes withdrawal of cortical cholinergic boutons in the adult rat. Proc Natl Acad Sci U S A 96:4067-4072.

Ebendal T (1992) Function and evolution in the NGF family and its receptors. J Neurosci Res 32:461-470.

Fahnestock M, Michalski B, Xu B, Coughlin MD (2001) The precursor pronerve growth factor is the predominant form of nerve growth factor in brain and is increased in Alzheimer's disease. Mol Cell Neurosci $18: 210-220$.

Fahnestock M, Yu G, Michalski B, Mathew S, Colquhoun A, Ross GM, Coughlin MD (2004) The nerve growth factor precursor proNGF exhibits neurotrophic activity but is less active than mature nerve growth factor. J Neurochem 89:581-592.

Figueiredo BC, Skup M, Bedard AM, Tetzlaff W, Cuello AC (1995) Differential expression of $\mathrm{p} 140^{\text {trk }}, \mathrm{p} 75^{\mathrm{NGFR}}$ and growth- associated phosphoprotein-43 genes in nucleus basalis magnocellularis, thalamus and adjacent cortex following neocortical infarction and nerve growth factor treatment. Neuroscience 68:29-45.

Gauthier S (1999) Acetylcholinesterase inhibitors in the treatment of Alzheimer's disease. Expert Opin Investig Drugs 8:1511-1520.

Giacobini E (1987) Modulation of brain acetylcholine levels with cholinesterase inhibitors as a treatment of Alzheimer disease. Keio J Med 36:381-391

Goedert M, Fine A, Hunt SP, Ullrich A (1986) Nerve growth factor mRNA in peripheral and central rat tissues and in the human nervous system: lesion effects in the rat brain and levels in Alzheimer's disease. Brain Res 387:85-92.

Greferath U, Bennie A, Kourakis A, Bartlett PF, Murphy M, Barrett GL (2000) Enlarged cholinergic forebrain neurons and improved spatial learning in p75 knockout mice. Eur J Neurosci 12:885-893.

Grimes ML, Zhou J, Beattie EC, Yuen EC, Hall DE, Valletta JS, Topp KS, LaVail JH, Bunnett NW, Mobley WC (1996) Endocytosis of activated TrkA: evidence that nerve growth factor induces formation of signaling endosomes. J Neurosci 16:7950-7964.

Hefti F, Knusel B, Lapchak PA (1993) Protective effects of NGF and BDNF on basal forebrain cholinergic neurons in adult rats with partial fimbrial transections. Prog Brain Res 98:257-263.

Hu L, Côté SL, Cuello AC (1997) Differential modulation of the cholinergic phenotype of the nucleus basalis magnocellularis neurons by applying NGF at the cell body or cortical terminal fields. Exp Neurol 143:162-171.

Hu L, Wong TP, Côté SL, Bell KF, Cuello AC (2003) The impact of Abetaplaques on cortical cholinergic and non-cholinergic presynaptic boutons in alzheimer's disease-like transgenic mice. Neuroscience 121:421-432.

Huang ZB, Wang H, Rao XR, Zhong GF, Hu WH, Sheng GQ (2011) Different effects of scopolamine on the retrieval of spatial memory and fear memory. Behav Brain Res 221:604-609. 
Kraemer PJ, Brown RW, Baldwin SA, Scheff SW (1996) Validation of a single-day Morris Water Maze procedure used to assess cognitive deficits associated with brain damage. Brain Res Bull 39:17-22.

Lee R, Kermani P, Teng KK, Hempstead BL (2001) Regulation of cell survival by secreted proneurotrophins. Science 294:1945-1948.

Leon WC, Bruno MA, Allard S, Nader K, Cuello AC (2010) Engagement of the PFC in consolidation and recall of recent spatial memory. Learn Mem 17:297-305.

Majdi M, Ribeiro-da-Silva A, Cuello AC (2007) Cognitive impairment and transmitter-specific pre- and postsynaptic changes in the rat cerebral cortex during ageing. Eur J Neurosci 26:3583-3596.

Mizoguchi H, Nakade J, Tachibana M, Ibi D, Someya E, Koike H, Kamei H, Nabeshima T, Itohara S, Takuma K, Sawada M, Sato J, Yamada K (2011) Matrix metalloproteinase- 9 contributes to kindled seizure development in pentylenetetrazole-treated mice by converting pro-BDNF to mature BDNF in the hippocampus. J Neurosci 31:12963-12971.

Morris RG, Garrud P, Rawlins JN, O’Keefe J (1982) Place navigation in rats with hippocampal lesions. Nature 297:681-683.

Mufson EJ, Counts SE, Fahnestock M, Ginsberg SD (2007) Cholinotrophic molecular substrates of mild cognitive impairment in the elderly. Curr Alzheimer Res 4:340-350.

Nykjaer A, Lee R, Teng KK, Jansen P, Madsen P, Nielsen MS, Jacobsen C, Kliemannel M, Schwarz E, Willnow TE, Hempstead BL, Petersen CM (2004) Sortilin is essential for proNGF-induced neuronal cell death. Nature 427:843-848.

Pang PT, Teng HK, Zaitsev E, Woo NT, Sakata K, Zhen S, Teng KK, Yung WH, Hempstead BL, Lu B (2004) Cleavage of proBDNF by tPA/plasmin is essential for long-term hippocampal plasticity. Science 306:487-491.

Paxinos G, Watson C (2005) The rat brain in stereotaxic coordinates, fifth edition. Sydney, Academic.

Peng S, Wuu J, Mufson EJ, Fahnestock M (2004) Increased proNGF levels in subjects with mild cognitive impairment and mild Alzheimer disease. J Neuropathol Exp Neurol 63:641-649.

Perry EK, Tomlinson BE, Blessed G, Bergmann K, Gibson PH, Perry RH (1978) Correlation of cholinergic abnormalities with senile plaques and mental test scores in senile dementia. Br Med J 2:1457-1459.

Ribeiro-da-Silva A, Priestley JV, Cuello AC (1993) Pre-embedding ultrastructural immunocytochemistry. In: Immunohistochemistry II (Cuello AC, ed), pp 181-227. Chichester, UK: Wiley.

Rogers SL, Friedhoff LT (1996) The efficacy and safety of donepezil in patients with Alzheimer's disease: results of a US multicentre, randomized, double-blind, placebo-controlled trial. The Donepezil Study Group. Dementia 7:293-303.

Sarter M, Parikh V, Howe WM (2009) Phasic acetylcholine release and the volume transmission hypothesis: time to move on. Nat Rev Neurosci 10:383-390.

Smith MA, Zhang LX, Lyons WE, Mamounas LA (1997) Anterograde transport of endogenous brain-derived neurotrophic factor in hippocampal mossy fibers. Neuroreport 8:1829-1834.

Sobreviela T, Clary DO, Reichardt LF, Brandabur MM, Kordower JH, Mufson EJ (1994) TrkA-immunoreactive profiles in the central nervous system: colocalization with neurons containing p75 nerve growth factor receptor, choline acetyltransferase, and serotonin. J Comp Neurol 350:587-611.

Turrini P, Casu MA, Wong TP, De Koninck Y, Ribeiro-da-Silva A, Cuello AC (2001) Cholinergic nerve terminals establish classical synapses in the rat cerebral cortex: synaptic pattern and age-related atrophy. Neuroscience 105:277-285.

Ullal GR, Michalski B, Xu B, Racine RJ, Fahnestock M (2007) NT-3 modulates BDNF and proBDNF levels in naive and kindled rat hippocampus. Neurochem Int 50:866-871.

Venero JL, Knüsel B, Beck KD, Hefti F (1994) Expression of neurotrophin and trk receptor genes in adult rats with fimbria transections: effect of intraventricular nerve growth factor and brain-derived neurotrophic factor administration. Neuroscience 59:797-815.

Veng LM, Granholm AC, Rose GM (2003) Age-related sex differences in spatial learning and basal forebrain cholinergic neurons in F344 rats. Physiol Behav 80:27-36.

Volosin M, Song W, Almeida RD, Kaplan DR, Hempstead BL, Friedman WJ (2006) Interaction of survival and death signaling in basal forebrain neurons: roles of neurotrophins and proneurotrophins. J Neurosci 26:7756-7766.

von Bartheld CS, Byers MR, Williams R, Bothwell M (1996) Anterograde transport of neurotrophins and axodendritic transfer in the developing visual system. Nature 379:830-833.

von Bartheld CS, Wang X, Butowt R (2001) Anterograde axonal transport, transcytosis, and recycling of neurotrophic factors: the concept of trophic currencies in neural networks. Mol Neurobiol 24:1-28.

Williams B, Nelson M, Granholm AC, Coultrap S, Browning M, Curtis M (2005) Altered NGF response but not release in the aged septohippocampal cholinergic system. Exp Neurol 196:30-40.

Wong TP, Debeir T, Duff K, Cuello AC (1999) Reorganization of cholinergic terminals in the cerebral cortex and hippocampus in transgenic mice carrying mutated presenilin-1 and amyloid precursor protein transgenes. J Neurosci 19:2706-2716.

Yang J, Siao CJ, Nagappan G, Marinic T, Jing D, McGrath K, Chen ZY, Mark W, Tessarollo L, Lee FS, Lu B, Hempstead BL (2009) Neuronal release of proBDNF. Nat Neurosci 12:113-115. 